\title{
Uso de macro dosis de oxitocina en mujeres con óbito fetal ${ }^{1}$
}

Institución: Universidad de Costa Rica

\author{
Ana Marcela Hernández González ${ }^{2}$ \\ Fabiana Jiménez Calderón ${ }^{3}$
}

\begin{abstract}
RESUMEN
El objetivo de este estudio fue analizar la mejor evidencia científica disponible sobre el uso de macro dosis de oxitocina comparado con dosis estándar en mujeres que presentaron óbito fetal en el año 2017. Las acciones profesionales obstétricas para la atención en los partos varían ampliamente entre los diferentes países, entre las diferentes instituciones nacionales y los diferentes equipos encargados de dicha atención. El realizar investigación en el campo de obstetricia nos permite determinar cuáles son las mejores acciones basadas en la evidencia científica y de esta manera ofrecer una atención de calidad a la usuaria gestante.

Para realizar el estudio se aplicó la metodología de Práctica Clínica Basada en la Evidencia; se inició con la elaboración de la pregunta clínica seguido por búsqueda de información en las bases de datos científicas como MEDLINE, COCHRANE, SCIENCIE DIRECT, TRIPDATA BASE, SCIELO y PUBMED. La búsqueda en todas las bases de datos recuperó un total de 2548 artículos, los cuales fueron revisados cuidadosamente por título y abstract. Al finalizar, se recuperó un artículo el cual fue objeto de análisis crítico. Se concluye que la mejor evidencia disponible es insuficiente para dar respuesta fiable a la pregunta clínica establecida sobre el tema.
\end{abstract}

Palabra clave: Muerte-fetal; Óbito-fetal; Oxitocina.

\footnotetext{
${ }^{1}$ Fecha de recepción: 20 de agosto del 2017

Fecha de aceptación: 27 de noviembre del 2017

2 Enfermera. Máster en Enfermería Ginecológica, Obstétrica y Perinatal. Hospital San Rafael de Alajuela. Costa Rica. Correo electrónico: marcela.hernandez.gonzalez@gmail.com

${ }^{3}$ Enfermera. Máster en Enfermería Ginecológica, Obstétrica y Perinatal. Universidad de Costa Rica. Costa Rica. Correo electrónico: fabijimenez27@gmail.com
} 


\title{
Use of macro doses of oxytocin in women with stillbirth ${ }^{1}$
}

Institution: University of Costa Rica

\author{
Ana Marcela Hernández González ${ }^{2}$ \\ Fabiana Jiménez Calderón ${ }^{3}$
}

\begin{abstract}
The objective of this study was to analyze the best available scientific evidence on the use of macro doses of oxytocin compared to standard doses in women who presented fetal death in the year 2017. The professional obstetric actions for delivery care vary widely among the different countries, between the different national institutions and the different teams responsible for such care. Conducting research in the field of obstetrics allows us to determine what are the best actions based on scientific evidence and thus provide quality care to the pregnant user.

In order to carry out the study, the methodology of Clinical Practice Based on Evidence was applied; It began with the elaboration of the clinical question followed by the search of information in the scientific databases such as MEDLINE, COCHRANE, SCIENCIE DIRECT, TRIPDATA BASE, SCIELO and PUBMED. The search in all the databases retrieved a total of 2548 articles, which were carefully reviewed by title and abstract. At the end, an article was retrieved which was the subject of critical analysis. It is concluded that the best available evidence is insufficient to give a reliable answer to the clinical question established on the subject.
\end{abstract}

Keywords: Fetal-death; Oxytocin; Stillbirth.

\footnotetext{
${ }^{1}$ Date of receipt: August 20, 2017

Date of acceptance: November 27, 2017

${ }^{2}$ Nurse. Master in Gynecological, Obstetric and Perinatal Nursing. San Rafael de Alajuela Hospital. Costa Rica. E-mail: :marcela.hernandez.gonzalez@gmail.com

${ }^{3}$ Nurse. Master in Gynecological, Obstetric and Perinatal Nursing. San Rafael de Alajuela Hospital. Costa Rica. E-mail: fabijimenez27@gmail.com
} 


\title{
Uso de macro doses de oxitocina em mulheres com óbito fetal ${ }^{1}$
}

Instituição: Universidade da Costa Rica

\author{
Ana Marcela Hernández González ${ }^{2}$ \\ Fabiana Jiménez Calderón ${ }^{3}$
}

\section{RESUMO}

O objetivo deste estudo foi analisar as melhores evidências científicas disponíveis sobre o uso de macro doses de oxitocina em comparação com doses padrão em mulheres que apresentaram morte fetal no ano 2017. As ações obstétricas profissionais para atendimento de parto variam amplamente entre as diferentes países, entre as diferentes instituições nacionais e as diferentes equipes responsáveis por esses cuidados. A realização de pesquisas no campo da obstetrícia nos permite determinar quais são as melhores ações baseadas em evidências científicas e, portanto, fornecer cuidados de qualidade para o usuário grávida.

Para realizar o estudo, foi aplicada a metodologia da Prática Clínica Baseada na Evidência; Começou com a elaboração da questão clínica seguida pela busca de informações nas bases de dados científicas, como MEDLINE, COCHRANE, SCIENCIE DIRECT, TRIPDATA BASE, SCIELO e PUBMED. A pesquisa em todos os bancos de dados recuperou um total de 2548 artigos, que foram cuidadosamente revisados por título e resumo. No final, um artigo foi recuperado, que foi objeto de análise crítica. Conclui-se que a melhor evidência disponível é insuficiente para dar uma resposta confiável à questão clínica estabelecida no assunto.

Palavras chave: Morte-fetal; Oxitocina; Óbito-fetal.

\footnotetext{
${ }_{1}^{1}$ Data de recepção: 20 de agosto del 2017

Data de aceitação: 27 de novembro de 2017

${ }^{2}$ Enfermeira. Mestre em Enfermagem Ginecológica, Obstétrica e Perinatal. Hospital de San Rafael de Alajuela. Costa Rica. Correio eletrônico: marcela.hernandez.gonzalez@gmail.com

${ }^{3}$ Enfermeira. Mestre em Enfermagem Ginecológica, Obstétrica e Perinatal. Hospital de San Rafael de Alajuela. Costa Rica. Correio eletrônico: fabijimenez27@gmail.com
} 


\section{Revista Electrónica Enfermeria Actual en costa Rica}

\section{INTRODUCCIÓN}

El embarazo y el parto son procesos complejos que experimenta una mujer para culminar con el nacimiento de un nuevo ser humano; sin embargo, cada una puede presentar situaciones diferentes durante este proceso que pueden arriesgar su salud y la de su bebé; tal situación, apela a los profesionales en obstetricia a determinar las acciones basadas en conocimiento científico para atender las diferentes necesidades que presente cada mujer en estas etapas de complejidad, lo cual incidirá en la salud de la mujer y del infante.

En torno al tema, Weeks ${ }^{1}$ señala que en el proceso de embarazo surgen situaciones fisiológicas que pueden complicar el desarrollo embrionario o fetal y afectar su desarrollo y crecimiento aunque, lamentablemente, en algunas ocasiones finaliza en un aborto o un óbito fetal. Ante este contexto surgen una serie de situaciones alrededor de la mujer y su situación de salud acerca de las que el profesional de enfermería obstétrica tiene la responsabilidad de intervenir en el cuidado de la salud de manera integral para prepararla al en el proceso de finalización de su embarazo. Al respecto, Ladewing et $\mathrm{al}^{2}$ mencionan que el método más utilizado para llevar a cabo la inducción o conducción de un parto es a través del uso de mecanismos farmacológicos.

En cuanto al uso de estos métodos farmacológicos, el que principalmente se administra es la oxitocina, un agente farmacológico utilizado en los procesos de inducción y conducción de un parto, cuyo fin es estimular el músculo liso del útero e, incrementar su excitabilidad y actividad para favorecer la propagación de las contracciones que se requieren para expulsar el feto dentro del proceso de parto. Este fármaco lo administra el profesional en ginecoobstetricia para vigilar el proceso y su evolución.

Una de las complicaciones que provoca la hiperestimulación uterina inducida por este fármaco es una ruptura de útero $\mathrm{y}$, secundariamente, una hemorragia que puede provocar la muerte materna, la cual puede ocurrir en periodos diferentes de atención, ya sea "durante el embarazo, parto o dentro de los 42 días después de terminación del mismo", tal lo define la OMS.

Tras realizar una búsqueda de artículos científicos e investigaciones a nivel mundial, no se ha encontrado estudios específicos que comparen la administración de dosis altas y estándares de oxitocina en el manejo de mujeres con óbito fetal; sin embargo, hay estudios que evidencian que las altas dosis de oxitocina no mejoran el proceso del parto y provocan hiperestimulación uterina, aumento de hemorragias postparto, fiebre, endometritis.

Entre algunos de estos estudios está el de Reyna et $\mathrm{al}^{3}$, en la Universidad de los Andes, Venezuela, quienes desarrollaron un estudio en mujeres que se sometieron a la finalización del embarazo durante el segundo trimestre, a causa de muerte fetal. Emplearon como métodos de inducción la oxitocina y el misoprostol, ambos grupos no presentaban dilatación cervical ni contracciones uterinas en el momento. El objetivo fue comparar la efectividad de ambos medicamentos; no obstante, los datos arrojados indicaron que el tiempo desde el inicio del tratamiento y la expulsión del feto fue más corto en las mujeres tratadas con misoprostol en comparación con la oxitocina; por otra parte, las complicaciones identificadas en el estudio fueron la hemorragia posparto, y la retención placentaria y efectos adversos como náuseas, vómitos y fiebre.

Por su parte, Wei et $\mathrm{al}^{4}$ mencionan cómo llevaron a cabo una revisión sistemática a partir de búsquedas en PubMed, Medline, Embase y Cochrane Library para ensayos clínicos aleatorios, cuyo objetivo fue determinar la 
eficacia y seguridad de dosis altas de oxitocina vs. dosis bajas en el aumento de la labor de parto. Concluyeron que la dosis alta de oxitocina está asociada con una disminución moderada en el riesgo de cesárea, disminuye la duración del parto, hay aumento de riesgo de hiperestimulación, pero no se evidenció aumento en la mortalidad materna o neonatal.

Respecto de las dosis altas de oxitocina y el riesgo de complicaciones maternas, Grotegut et $\mathrm{al}^{5}$ mencionan en su estudio realizado en Orlando Florida, de casos y controles cuyo objetivo fue determinar si las mujeres que presentaron hemorragia posparto severa a causa de atonía uterina, recibieron altas dosis de oxitocina. El estudio calculó el total de exposición a oxitocina como el área bajo la curva de concentración (mU/min*min), sobre lo que se concluyó que las mujeres con hemorragia posparto severa secundaria a atonía uterina fueron expuestas significativamente a mayor oxitocina durante la labor de parto, comparado con el grupo control.

En la investigación realizada por Singh et $\mathrm{al}^{6}$, en el 2015, del Departamento de Ginecología y Obstetricia, en India, los autores; publicaron Ruptura uterina: todavía una dura realidad, un estudio relacionado con el uso y administración de la oxitocina, cuyo objetivo fue determinar la incidencia, etiología, manejo, resultado materno y fetal, y evaluar tendencias en el área y recomendar medidas prevenibles. Se llevó a cabo un estudio transversal en mujeres que presentaron ruptura uterina de enero del 2012 a agosto del 2013. Como dato relevante, los investigadores describen que dentro de los principales factores de riesgo encontrados fue el uso desinteresado de la oxitocina $(52,5 \%)$ con una mortalidad perinatal del $85 \%$ y una muerte materna $(2,5 \%)$.

En el ámbito nacional no se encontró estudios que respalden la administración de macrodosis o dosis estándar de oxitocina en mujeres que presentan un óbito fetal; a pesar de que esta práctica se lleva a cabo en los servicios de maternidad de hospitales nacionales.

Ante este panorama surge la interrogante sobre los criterios con los que se establece la dosis de oxitocina y su frecuencia durante la atención de la mujer con una muerte intrauterina, así como la obtención de la mejor evidencia disponible al respecto.

Para responder, Ladewing, et $\mathrm{al}^{7}$ indica que un profesional en enfermería debe ofrecer calidad en la atención y servicios, brindando asistencia integral a las usuarias, así como cambios en la práctica obstétrica, dependiendo de su formación, preparación y especialización.

Los profesionales en obstetricia deben manejar ampliamente las complicaciones que pueden afectar la salud fetal, para que de esta manera pueda ofrecer una atención especializada en los diferentes niveles de atención y tener conocimiento de las múltiples complicaciones que se pueden presentar; al respecto, Schwarcz et al ${ }^{8}$ mencionan que "las causas de muerte fetal son múltiples de origen ambiental, materno, ovular y fetal y en parte desconocidas",

Tras analizar la importancia del tema y la relevancia en los centros de salud y los cambios en la atención que reciben las usuarias con muerte fetal intrauterina es que se da la descripción de la pregunta clínica y el objetivo general mediante los cuales se desarrolló esta investigación. 
¿En mujeres que presentan un óbito fetal, la aplicación de macro dosis de oxitocina para la inducción del parto comparado con la de oxitocina a dosis estándar aumenta el riesgo de complicaciones y mortalidad materna?

Con base en esta pregunta de investigación, se realizó la investigación con el objetivo de analizar la mejor evidencia científica disponible respecto de la aplicación de macro dosis de oxitocina y el riesgo de complicaciones y mortalidad materna comparada con la oxitocina a dosis estándar para la inducción de mujeres que presentan un óbito fetal.

\section{MATERIALES Y MÉTODOS}

Esta investigación es basada en la evidencia; la cual, como lo refiere Ingersoll, mencionado por Alcolea et al $^{9}$ es "el uso consciente, explícito y juicioso crítico de información derivada de la teoría y basada en la investigación para la toma de decisiones sobre prestación de cuidados a sujetos o grupos, teniendo en cuenta sus preferencias y necesidades". 9

A pesar de que la enfermería basada en la evidencia, ha sido una rama de la investigación que se ha venido implantando desde hace ya más de dos décadas, no todos los profesionales se basan en este importante recurso como fuente de conocimiento sobre el cuidado obstétrico. En este sentido, es importante mencionar que al incorporarse en el desempeño la investigación basada en la evidencia, para realizar el cuidado obstétrico, las enfermeras ginecoobstetras, a la vez, se involucran con la práctica basada en la evidencia, adoptando, como lo menciona Alcolea et al "los conocimientos generados por la investigación científica de calidad, incorporando la experiencia profesional, las demandas y valores de los usuarios y los recursos existentes. Como consecuencia de ello, las decisiones que deben tomar los profesionales para elegir el mejor cuidado para su paciente concreto, al verse apoyadas en estos elementos, conducen a una menor variabilidad en la práctica clínica y trabajar con práctica basada en la evidencia va formando parte de la cultura de calidad de la atención sanitaria que prestamos a nuestros pacientes."

La enfermería basada en la evidencia, está respaldada por las siguientes etapas o fases importantes que permitirán concretar la respuesta que busca el investigador.

Las siguientes fases son expuestas por los autores Alcolea et $\mathrm{al}^{9}$ :

Fase 0 o spirit of inquiry: es la primera etapa de la investigación, inicia cuando se presenta a través una interrogante, Melnyk (2009), citado por Alcolea et al ${ }^{9}$ y lo llama "spirit of inquiry", sobre lo que dichos autores comentan que "es, el surgimiento de una inquietud que conduce a interrogarse acerca del porqué de los cuidados prestados a los pacientes en su realidad cotidiana". En relación con lo anterior, en esta etapa de investigación surgió la interrogante en torno a conocer el manejo de la mujer que experimenta muerte fetal, así como del tratamiento más apropiado, por lo tanto, se preguntó a profesionales obstétricos de diferentes centros hospitalarios para conocer sobre el uso de la oxitocina a altas dosis para la inducción del parto, lo cual, de acuerdo con las consultas realizadas, hay dudas sobre el fundamento científico de dicha práctica, así como de la relación de efectos adversos indeseables en la mujer que se somete a esta intervención, entre los que se incluye el riesgo de mortalidad. 
Fase 1 o formulación de la pregunta clínica: Posterior al planteamiento de la inquietud, la literatura refiere que se debe continuar con la elaboración de la pregunta de investigación ( para efectos de la Práctica Basada en la Evidencia se llama pregunta clínica). Para elaborar esta pregunta, los autores plantearon un método concreto, conocido con el acrónimo PICO (en lengua inglesa): P (pacientes o problema a estudio) I (intervención) C (comparación), O (resultados-outcomes, en inglés).

Específicamente, en esta investigación, la pregunta clínica fue la siguiente ¿En mujeres que presentan un óbito fetal, la aplicación de macrodosis de oxitocina comparado con dosis estándar aumenta el riesgo de mortalidad materna? Partiendo de lo mencionado, el acrónimo PICO se desglosa de la siguiente forma P: mujeres que presentan un óbito fetal; I: aplicación de macrodosis de oxitocina; C: aplicación de oxitocina a dosis estándar para la inducción del parto; O: aumento de riesgo de complicaciones y mortalidad materna.

Una vez elaborada estructuralmente la pregunta clínica, se debe definir el diseño del estudio acorde con la investigación. En este estudio, se busca encontrar los efectos potenciales durante el uso de dosis elevadas de oxitocina que podrían constituir un riesgo perjudicial para la mujer con óbito fetal durante la inducción del parto; por tanto, de acuerdo con la literatura, evidenció una pregunta de daño, para la que se recomienda, según Alcolea et $\mathrm{al}^{9}$ que la selección de estudios debe de ser de tipo ensayos clínicos controlados y aleatorizados, estudios de cohortes, casos y controles y series de caso, revisiones sistemáticas.

Fase 2 o búsqueda de la evidencia científica: En esta fase se debe elaborar descriptores con los que se va a realizar búsqueda de la investigación. Se requiere varios elementos como las bases de datos a las que se recurra, el tipo de estudios que estas contengan, los descriptores utilizados, habilidades del investigador, lo cual subraya la importancia de una adecuada elaboración de la fase anterior.

En el presente estudio, los descriptores determinados que forman el acrónimo PICO como términos claves para el desarrollo de la búsqueda son óbito fetal, inducción del parto en óbito fetal, macrodosis de oxitocina, dosis estándar de oxitocina, muerte materna, complicaciones maternas. Una vez determinados los descriptores, se procede a establecer las relaciones entre estos, así como su traducción al inglés y portugués.

Continuando con esta fase, se define los criterios de inclusión para recolectar la información: es esta etapa se considera la población, constituida por mujeres embarazadas de todas las edades, ya sea nulíparas o multíparas, con o sin algún antecedente patológico que presentan una muerte fetal. El tiempo en que se desarrolla búsqueda de la información que corresponde al periodo comprendido entre los años 2012 y 2017 . Dado el tipo de estudio, y considerando que la pregunta clínica evidencia ser una pregunta de "daño", se incluyó de acuerdo con las recomendaciones de la literatura ensayos clínicos controlados y aleatorizados, estudios de cohortes, casos y controles y revisiones sistemáticas. En cuanto al idioma, se limitó a español, inglés y portugués.

Una vez definido lo anterior, se procedió a establecer la relación de los descriptores, como parte de esta fase, para lo que es indispensable aplicar los operadores booleanos los cuales, de acuerdo con Muñoz et al ${ }^{10}$, toda base de datos reconoce tres, (AND), O (OR), O (NOT):cada uno delimita o amplía la búsqueda de la información según las necesidades del investigador. 
A continuación, se definen las relaciones establecidas en este estudio, véase tabla 1.

Tabla 1. Presentación de los descriptores.

SEP/ Maestría Enfermería GOP. Presentación de los descriptores, 2017

\begin{tabular}{|l|l|l|}
\hline \multicolumn{1}{|c|}{ DESCRIPTOR } & OPERADOR BOOLEANO & \multicolumn{1}{c|}{ DESCRIPTOR 2 } \\
\hline 1 Induction of delivery in fetal death & AND & Oxytocin macrodosis infusion \\
\hline $\begin{array}{l}\text { 2. Induction of delivery in fetal death } \\
\text { 3. Stillbirth }\end{array}$ & AND & Standard Oxytocin Dosage \\
\hline 4. Stillbirth & AND & Oxytocin macrodosis infusion \\
\hline 5. Oxytocin macrodosis & AND & Standard dose oxytocin. \\
\hline 6. Oxytocin macrodosis & INCREASES & Maternal Complications \\
\hline 7. Oxytocin Macrodosis & DOES NOT INFLUENCE & Maternal Complications \\
\hline 8. Oxytocin macrodosis & INCREASES RISK & Maternal Mortality \\
\hline 9. Standard Oxytocin Dosage & DOES NOT INFLUENCE & Maternal Mortality \\
\hline 10. Standard Oxytocin dose & DOES NOT INFLUENCE & Maternal Complications \\
\hline 11. Oxytocin Macrodosis & USED IN & $\begin{array}{l}\text { Induction of delivery in fetal } \\
\text { death }\end{array}$ \\
\hline
\end{tabular}

Fuente: elaboración propia

Fase 3 o valoración crítica de la evidencia: es una etapa que conlleva un exhaustivo rigor en la lectura crítica de los estudios seleccionados y que implica para el investigador la adquisición de destrezas y habilidades indispensables.

Además, González et $\mathrm{al}^{11}$ mencionan que en esta etapa es necesario desarrollar destrezas para valorar los documentos que se encuentran en el periodo de búsqueda de información, por lo que es importante determinar calidad, veracidad, importancia, aplicabilidad. Para este estudio se implementó la plataforma electrónica FLC 2.0, una aplicación que permite agilizar la lectura, sintetizar la evidencia encontrada, evaluar los diseños de investigación. Se utilizó además el instrumento PRISMA el cual permite evaluar la calidad y la evidencia sistemática encontrada en el periodo de la investigación.

Fase 4. Transferencia de la evidencia a la práctica: en esta etapa se elaboran instrumentos o acciones para realizar actividades de divulgación de los resultados y a partir de ello determinar cambios en la práctica clínica, con el fin de mejorar la atención de los usuarios a quienes se dirige la investigación. Al respecto, Alcolea et al la definen como "la transmisión de los resultados de la investigación a las instituciones, profesionales sanitarios y pacientes con el objetivo de propiciar, si es preciso, cambios en la práctica clínica"9.

En lo que respecta a la presente investigación para llevar a cabo esta fase, se divulgó los resultados a través de la evidencia por medio de un boletín que contiene la síntesis de los apartados de este manuscrito, el cual se ofreció de forma gratuita a los obstetras que laboran en las maternidades de siete instituciones de salud del país en la Caja Costarricense de Seguro Social (Hospital Adolfo Carit Eva, Hospital Dr. Rafael Ángel Calderón Guardia, 
Hospital México, Hospital San Vicente de Paúl, Hospital Max Peralta de Cartago, Hospital San Rafael de Alajuela, Hospital San Juan de Dios; y dos hospitales privados que ofrecen servicio de maternidad en el país: Hospital Clínica Bíblica y Hospital La Católica).

Fase 5. Evaluación de los resultados de la utilización de la evidencia: la última fase corresponde a la evaluación de los resultados a través de la evidencia. Específicamente en esta investigación, posterior a la transmisión de los resultados, a los profesionales de enfermería ginecoobstétrica del tercer nivel de atención les corresponderá implementar las recomendaciones a partir de los resultados y ajustarlas al contexto en el que se desenvuelven, para así emitir un criterio de evaluación.

\section{Consideraciones éticas}

La ética en la práctica basada en la evidencia implica considerar los principios fundamentales como autonomía, beneficencia y no maleficencia, además de las consideraciones éticas que debe tener presente el investigador durante el desarrollo del estudio, tales como el plagio y los conflictos de intereses.

Autonomía: En relación con la autonomía, Cabero et $\mathrm{al}^{12}$ la definen como "proceso de atención que requiere como principio que se reconozca y se practiquen las referencias en el valor del paciente en función de sus creencias y valores". El primer enfoque contenido en la PBE es el reconocimiento del principio de autonomía, el cual incorpora los valores y preferencias de los usuarios en relación con la toma de decisiones sobre los cuidados que aplicará en respuesta a la evidencia obtenida en determinada situación. Debe existir una complementariedad en el ejercicio de este principio, tanto del profesional de salud como del usuario o usuaria, a quien se le debe de transmitir el conocimiento sobre la práctica por realizar, de manera que tome la decisión con base en las recomendaciones a la luz de la evidencia, lo cual concuerda con Siruana ${ }^{13}$ quien agrega que "la autonomía de una persona es respetada cuando se le reconoce el derecho a mantener puntos de vista, a hacer elecciones y a realizar acciones basadas en valores y creencias personales".

Beneficencia: el principio de la investigación y el análisis de prácticas se realiza con el objetivo de mejorar las acciones y los cuidados de la población con quien se desarrolla la investigación, así lo define De la Torre ${ }^{14}$ "la beneficencia debe centrarse en la relación riesgo-beneficio sin recaer en los límites", de manera que los resultados generen beneficios a la población ya sea cambiando, mejorando, o realizando prácticas seguras en la atención de los usuarios. En torno al concepto, Siruana ${ }^{13}$ indica que "consiste en prevenir el daño, eliminar el daño o hacer el bien a otros, mientras que la no-maleficencia implica la ausencia de acción, la beneficencia incluye siempre la acción".

No maleficencia: este principio se basa en que las acciones realizadas ante la atención o investigación no produzcan un daño físico o mental sobre un usuario, sobre el que Siruana 13 indica que "hace referencia a la obligación de no infligir daño intencionadamente"; es decir, no realizar daño en lo físico y mental que complemente a un individuo.

Plagio: de acuerdo con Redondo ${ }^{15}$, el plagio se presenta en algunos procesos de educación y de investigación en diferentes países e instituciones, el cual se define como una "apropiación de las ideas o palabras expresadas por otras personas en diferentes documentos o fuentes sin indicar su procedencia con el propósito normal de obtener alguna ventaja dentro de una institución”. Por su parte, Benos (2005) y Nayak (2006) citados por Laguna et al ${ }^{16}$ lo 


\section{Revista Electrónica Enfermeria Actual en costa Rica}

definen como "un apropiamiento de ideas, palabras o resultados de otras personas sin otorgarles el reconocimiento que se merecen." En torno a esta investigación, los artículos y documentos utilizados se mencionan con las normas establecidas: se utilizó el sistema de citas y referencias bibliográficas según normas Vancouver $2016^{17}$, determinadas por la Asociación Médica Británica (BMA).

Declaración de conflicto de intereses: en lo concerniente al conflicto de intereses, Selgales ${ }^{18}$ indica que existe uno cuando un autor (o la institución del autor), el revisor o el editor tienen relaciones financieras o personales que influyen impropiamente (sesgo) en sus acciones (tales relaciones se conocen también como compromisos duales, intereses en competencia, o lealtades competitivas). Para Benos (2005) y Nayak (2006) citados por Laguna et $\mathrm{al}^{16}$, "es cuando un autor, revisor o editor tienen relaciones personales o financieras que puedan influenciar de manera inapropiada en las acciones tomadas en el momento de su realización.”.

Por lo anterior, a partir de diciembre de 2011, se exige a los autores declarar conflictos de intereses al momento del envío de sus artículos, tanto para artículos originales, casos clínicos, imágenes en medicina, artículos de revisión, artículos especiales y cartas al editor.

\section{RESULTADOS}

La búsqueda de los resultados y su análisis, se llevó a cabo por dos revisores, durante marzo, abril y mayo del 2017, los cuales se incorporaron a la plataforma de FCL 2.0, usada para analizarlos, además de que se utilizó los grados de recomendación según la propuesta de Oxford.

La búsqueda de los artículos para llevar a cabo el análisis crítico fue realizada a través de cinco bases de datos Science Direct, Medline, Cochrane Library Plus, Tripdatabase, PubMed y Scielo. Se utilizó como filtros las publicaciones entre los años 2012-2017, con idioma inglés, español o portugués, las investigaciones sobre mujeres gestantes de todas las edades, nulíparas o multíparas, con o sin algún antecedente patológico que presentan una muerte fetal y los que incluían estudios de ensayos clínicos controlados y aleatorizados, estudios de cohortes, casos y controles. La figura 1 muestra el algoritmo de búsqueda. 
Figura 1. SEP/Maestría en Enfermería GOP: Algoritmo de búsqueda, resultados de acuerdo con los descriptores en cada base de datos, 2017

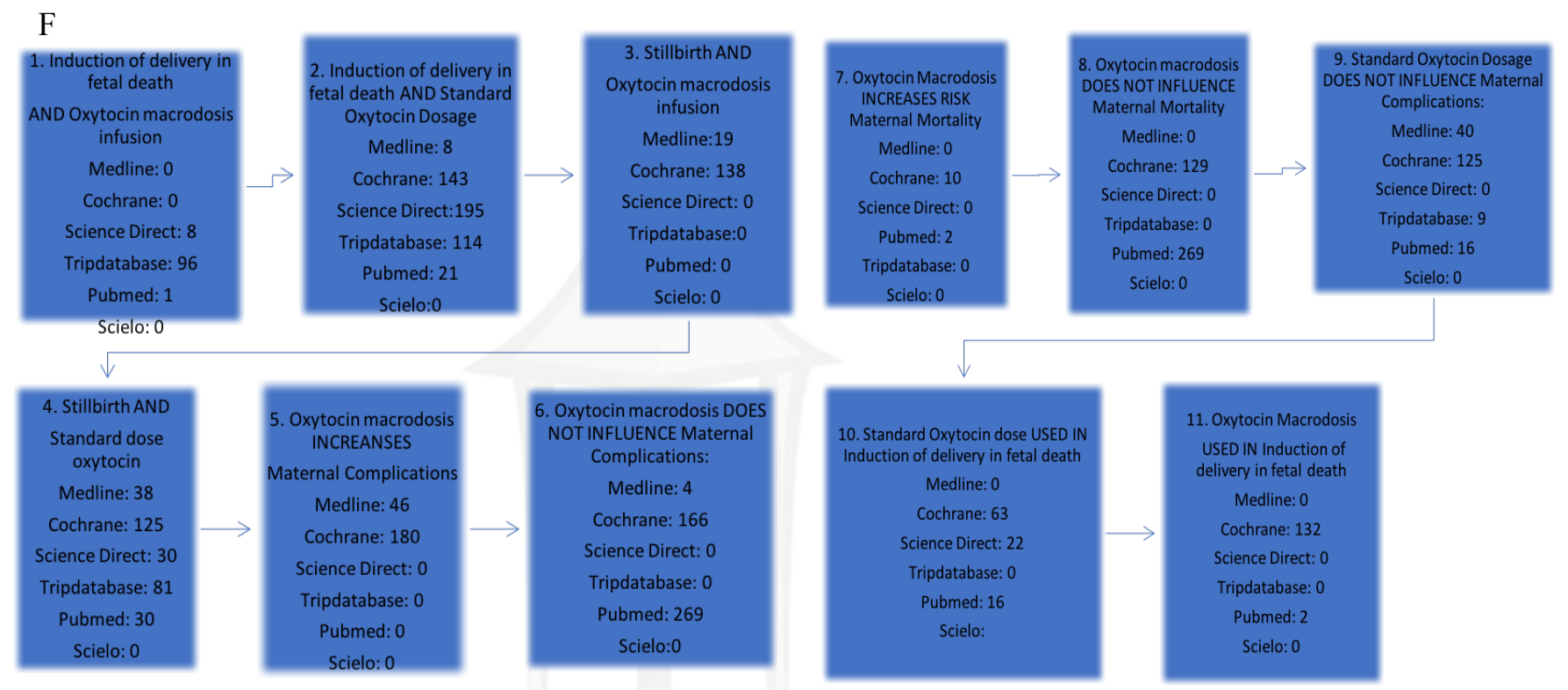

Fuente : Elaboración propia

Los descriptores que arrojaron mayor cantidad de resultados en las bases de datos seleccionados fueron Induction of delivery in fetal death AND Standard Oxytocin Dosage (481), Oxytocin macrodosis DOES NOT INFLUENCE Maternal Complications (439) y Oxytocin macrodosis DOES NOT INFLUENCE Maternal Mortality (398): en relación con estos dos últimos descriptores, en la información de estudios recopilados en la base de datos de PubMed, se obtuvo los mismos artículos, mientras que de Stillbirth AND Standard Dose Oxytocin, 304 resultados. Es importante aclarar que, a pesar de haberse incorporado en la metodología la traducción de los descriptores y búsqueda de los mismos en el idioma portugués, no hubo resultados al respecto, por tanto, solamente se presentan en inglés.

De acuerdo con los descriptores y las relaciones en todas las bases de datos, la búsqueda recobró un total de 2548 artículos, los cuales fueron introducidos a una serie de pasos para aproximar los estudios que no respondían a la pregunta clínica, los cuales se detalla a continuación.

Título: el tema o título de cada artículo encontrado brindó una primera aproximación a la respuesta de la pregunta clínica, por lo que en primer lugar se descartó aquellos que no brindaban una aproximación o no se relacionaban. A pesar de que se realizó una búsqueda exhaustiva con un total de 11 descriptores y seis bases de datos diferentes, la mayoría de los artículos encontrados no se aproximan directamente a responder la pregunta clínica planteada por las investigadoras, razón por la que se descartó 2519 artículos.

Abstract: se procedió a descartar once estudios según el resumen, tomando como referencia el resultado principal y la conclusión en cada uno de ellos, y si su contenido respondía a la pregunta clínica y a los objetivos trazados. 


\section{Revista Electrónica Enfermeria Actual en costa Rica}

Duplicación: este criterio se aplicó a artículos que no cumplían con los criterios de inclusión y que se encontraban duplicados en las diferentes bases de datos o en las mismas bases de datos, pero se clasificaron por diferentes descriptores. Al contrario, no cumplían con criterios de inclusión; sin embargo, anteriormente fueron descartados por abstract, por lo tanto, al aparecer nuevamente en una revisión posterior se tomó como duplicado. De acuerdo a lo arrojado en la búsqueda, se descartaron 17 estudios duplicados.

A continuación, en la tabla 2 se expone los resultados del proceso de selección y eliminación de artículos obtenidos en la búsqueda según cada base de datos.

Tabla 2. SEP/MaestriaMaestría Enfermería GOP. Proceso de descarte y selección según bases de datos, 2017.

\begin{tabular}{|l|c|c|c|c|}
\hline PROCESO DE DESCARTE Y SELECCIÓN & \multicolumn{2}{l|}{} \\
\hline Bases de datos & Título & Abstract & Duplicado & $\begin{array}{l}\text { Seleccionado para } \\
\text { análisis crítico }\end{array}$ \\
\hline Cochrane & 1209 & 0 & 2 & 1 \\
\hline Medline & 146 & 7 & 2 & 0 \\
\hline Science Direct & 253 & 0 & 2 & 0 \\
\hline Tripdatabase & 299 & 0 & 1 & 0 \\
\hline Pubmed & 612 & 4 & 10 & 0 \\
\hline Scielo & 0 & 0 & 0 & 1 \\
\hline Total & $\mathbf{2 5 1 9}$ & $\mathbf{1 1}$ & $\mathbf{1 7}$ & \\
\hline
\end{tabular}

Fuente: elaboración propia.

De los 2548 artículos extraídos en las cinco bases de datos, se descartó un total de 2519 por título, 11 por abstract (dentro deentre las razones de descarte fue por combinación de métodos de inducción en los estudios realizados, por muerte fetal y métodos de inducción, cuya metodología se basaba en revisión de literatura); se descartó 17 por duplicados en las bases de datos.

De modo que, como se muestra en la tabla 2, se seleccionó un artículo que fue analizado de forma crítica, obtenido a través de la base de datos de Cochrane, con el descriptor Stillbirth AND Oxytocin Macrodosis Infusion; dicho estudio fue encontrado también en TRIP Database con el descriptor Induction of delivery in fetal death AND Oxytocin macrodosis infusion:

Budden A, Chen LJY, Henry A19.19 High-dose versus low-dose oxytocin infusion regimens for induction of labour at term. Cochrane Database of Systematic Reviews 2014, Issue 10. Art. No.: CD009701. DOI: 10.1002/14651858.CD009701.pub2

Para ésta esta investigación, se utilizó la plataforma de fichas de lectura crítica, FLC 2.0, el artículo seleccionado corresponde a una revisión sistemática. En cuanto a la evaluación, se aplicó los criterios que plantea PRISMA (2009), de acuerdo con ésta, el artículo seleccionado cumple con los criterios requeridos; por lo tanto, se considera un estudio de alta calidad. 
Entre los elementos que se consideró para evaluar la calidad de los estudios, en el caso de revisión sistemática seleccionada, son los siguientes: Pregunta de investigación, diseño metodológico (criterios de inclusión y exclusión, búsqueda bibliográfica, calidad de los estudios y extracción de datos), resultados del proceso de búsqueda y selección, síntesis de la evidencia, resultados clínicos de la revisión, conclusiones del estudio, conflictos de interés, validez externa del estudio.

La última parte del análisis está conformada por la clasificación de la evidencia, según el nivel y el grado de recomendación, realizada con la Escala de Oxford. (Tabla 3).

El estudio seleccionado, a cargo de Budden, Chen y Henry, corresponde a una revisión sistemática, con diseños de ensayos controlados aleatorios y ensayos controlados cuasialeatorios, cuyo objetivo fue determinar, la efectividad y seguridad de las dosis altas y bajas de oxitocina para la inducción del parto a término (de 37 semanas completas de gestación en adelante) a partir de la mejor evidencia disponible.

En lo que respecta a la metodología del estudio, se llevó a cabo una búsqueda del registro de ensayos del grupo Cochrane de embarazo y parto el cual registró 44 informes, relativos a 38 estudios de los cuales se incluyó nueve: estos últimos, que involucraron a 2391 mujeres y sus bebés, fueron revisados por los autores. Todos los ensayos compararon el dar a las mujeres una dosis alta versus una dosis baja de oxitocina para la inducción del parto.

En los criterios de inclusión, además de los tipos de diseños de estudios seleccionados por los investigadores (ensayos controlados aleatorios y ensayos controlados cuasialeatorios), se incluyó a mujeres embarazadas para la inducción del trabajo de parto con un feto viable a partir de 37 semanas de gestación completa. De igual forma, se consideró aquellos diseños que compararon el protocolo de oxitocina para la inducción del trabajo de parto, en donde específicamente se utilizara la dosis alta de oxitocina de al menos $100 \mathrm{mU}$ en los primeros 40 minutos, con incrementos de al menos $600 \mathrm{mU}$ en las dos primeros horas, en comparación con la dosis baja de oxitocina, definida como menos de $100 \mathrm{mU}$ de oxitocina en los primeros 40 minutos, y los incrementos de entrega de menos de $600 \mathrm{mU}$ total en las dos primeras horas. Se excluyó los ensayos cruzados y los ensayos aleatorios por grupo.

En torno a la evaluación del riesgo de sesgo, se llevó a cabo por parte de dos revisores utilizando los criterios descritos en el Manual Cochrane para Revisiones Sistemáticas de Intervenciones. Se evaluó el método como bajo riesgo de sesgo (cualquier proceso verdaderamente aleatorio), alto riesgo de sesgo (proceso no aleatorio) y riesgo de sesgo poco claro. Se llevó a cabo por medio: del cegamiento de los participantes y del personal (comprobación de posibles sesgos de desempeño), cegamiento en la evaluación de los resultados (posibles sesgos de detección), datos incompletos de los resultados (verificación de posibles bajas de desgaste a través de retiros, deserciones, desviaciones del protocolo), sesgo de notificación selectiva y riesgo de sesgo en general. Los resultados se formularon a través de coeficientes de riesgo (RR) con intervalos de confianza del $95 \%$ en el caso de los datos dicotómicos y para los resultados continuos se utilizó la diferencia de medias en las que los resultados se midieron de la misma forma entre los ensayos.

En cuanto a los resultados del estudio, los autores los separan en resultados primarios y secundarios. Los primeros se basan en relación a entrega vaginal no lograda en 24 horas, en donde los autores exponen que no se revelaron diferencias significativas en las tasas de parto vaginal no logradas dentro de las 24 horas (RR 0,94 con un 
intervalo de confianza del 95\% (IC) 0,78 a 1,14 en dos ensayos con 1339 mujeres) o una cesárea (RR 0,96, IC del 95\%: 0,81 a 1,14, ocho lactantes, 2023 mujeres).

En la tabla 3 se refleja los datos obtenidos por la plataforma de lectura crítica, la evaluación de los puntos metodológicos que llevó a la selección del estudio con un nivel de evidencia alto y su análisis de acuerdo con los grados de recomendación de Oxford.

Tabla 3. SEP/ Maestría Enfermería GOP. Procesamiento de información de los documentos incluidos, para análisis crítico según la plataforma FLC 2.0, 2017.

\begin{tabular}{|c|c|c|c|c|c|c|c|c|}
\hline $\begin{array}{c}\text { Referencia } \\
\text { Cita abreviada }\end{array}$ & $\begin{array}{c}\text { Estudio } \\
\text { Objetivos }\end{array}$ & Población & Intervención & Comparación & $\begin{array}{c}\text { Resultados } \\
\text { N.o de estudios y pacientes }\end{array}$ & Conclusiones & Comentarios & Calidad de la evidencia \\
\hline $\begin{array}{c}\text { Budden A, et al. } \\
2014\end{array}$ & $\begin{array}{l}\text { Determinar la eficacia } \\
\text { y la seguridad de la } \\
\text { dosis alta y baja de } \\
\text { oxitocina para la } \\
\text { inducción del parto a } \\
\text { término }\end{array}$ & $\begin{array}{c}\text { Mujeres embarazadas } \\
\text { para la inducción del } \\
\text { trabajo de parto con un } \\
\text { feto viable a partir de } \\
37 \text { semanas de } \\
\text { gestación completa }\end{array}$ & $\begin{array}{c}\text { Alta dosis inicial y } \\
\text { continua de oxitocina } \\
\text { para la inducción del } \\
\text { parto }\end{array}$ & $\begin{array}{l}\text { Bajas dosis de } \\
\text { oxitocina }\end{array}$ & 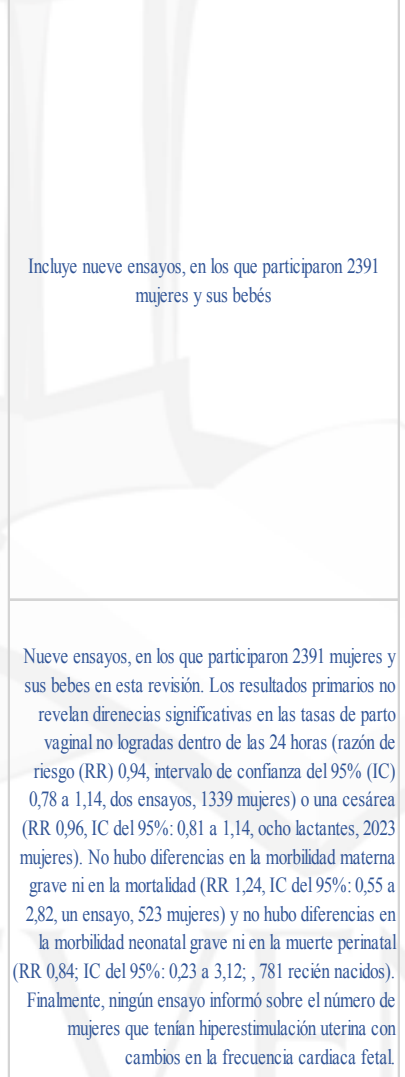 & 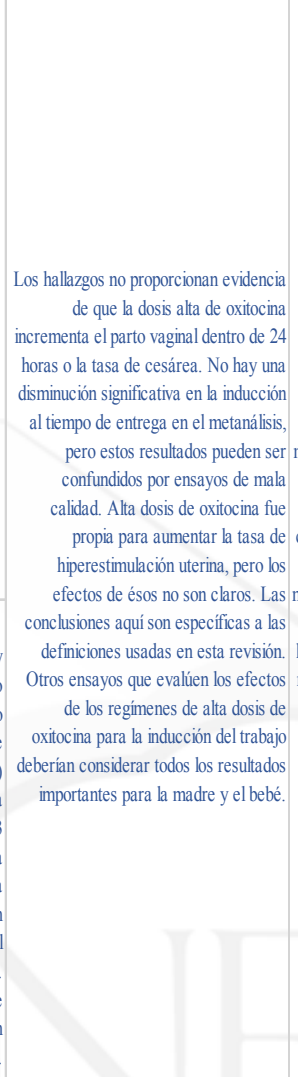 & $\begin{array}{l}\text { La dosis alta de oxitocina es } \\
\text { de al menos } 100 \mathrm{mU} \text { de } \\
\text { oxitocina en los primeros } 40 \\
\text { minutos, con incrementos de al } \\
\text { menos } 600 \mathrm{mU} \text { en los dos } \\
\text { primeros horas. En } \\
\text { comparación con la dosis baja } \\
\text { de oxitocina, definida como } \\
\text { menos de } 100 \mathrm{mU} \text { de oxitocina } \\
\text { en los primeros } 40 \text { minutos, y } \\
\text { los incrementos de entrega de } \\
\text { menos de } 600 \mathrm{mU} \text { total en las } \\
\text { dos primeras horas. }\end{array}$ & Alta \\
\hline
\end{tabular}

Fuente: Elaboración propia

En relación con lo anterior, como dato relevante, en cuanto a la morbilidad materna grave y mortalidad no se reportaron diferencias (RR 1,24; con un IC del 95\%: 0,55 a 2,82; en un ensayo con 523 mujeres). Los autores exponen que el estudio no arrojó diferencias en la morbilidad neonatal grave ni en la muerte perinatal (RR 0,84; con un IC del 95\%: 0,23 a 3,12; en 781 recién nacidos). Por último, en ninguno de los ensayos se reportó sobre el número de mujeres con hiperestimulación uterina con cambios en la frecuencia cardiaca fetal: sostienen que no observaron diferencias en los dos grupos (dosis altas vs. dosis bajas) en relación con complicaciones serias, como 
muerte materna o perinatal; sin embargo, el grupo que recibió dosis altas tuvo mayor riesgo de hiperestimulación uterina, de cuyos efectos no tienen claridad.

A pesar de los resultados revelados, los autores refieren que tanto el número de madres como bebés incluidos pudo haber sido pequeño para mostrar diferencia en resultados más específicos como la infección neonatal, síntomas maternos y desprendimiento placentario. Asimismo, afirman que la evidencia actual no es lo suficientemente fuerte, que apoye la administración de dosis altas sobre regímenes de dosis bajas de oxitocina para la inducción del parto, por ende, los autores recomiendan más investigaciones.

En torno a la calidad del artículo, se tomó en cuenta el desarrollo metodológico en diferentes aspectos, como el establecimiento de los criterios de selección, una búsqueda exhaustiva y rigurosa de los estudios, respecto de los que se estableció criterios de exclusión sobre el protocolo de la oxitocina definido en la revisión, además de la ausencia de conflictos de interés explícita en la investigación y la presentación de los resultados a través de un análisis riguroso de los mismos, por lo que se cataloga como un estudio de calidad alta.

\section{DISCUSIÓN}

En este estudio no se encontró evidencia científica que respalde la administración de macrodosis de oxitocina o dosis estándar para el manejo de una gestante con óbito fetal y las complicaciones que dicha intervención podría generar. El análisis se aborda de la siguiente manera: no hay suficiente evidencia que respalde el uso de macrodosis de oxitocina en óbito fetal: sin embargo, se encontró evidencia en embarazos viables.

Se debe indagar sobre la mejor evidencia científica disponible que respalde la aplicación de oxitocina a dosis estándar y el riesgo de complicaciones y mortalidad materna, en mujeres con óbito fetal.

A pesar de la frecuencia de nacimientos muertos (24 000 mil nacimientos muertos al año), en países como EE.UU., Boyel et al ${ }^{20}$ afirman que los datos sobre el manejo del parto son limitados, además de que previamente el uso de la oxitocina y las prostaglandinas, generalmente existe un manejo expectante. Los autores mencionados aseveran que aproximadamente un $75 \%$ de las mujeres con una muerte fetal iniciarán el trabajo de parto, dentro de las dos semanas del diagnóstico, así como que un estudio sobre la muerte fetal refieren que con la disponibilidad de fármacos como la oxitocina y prostaglandinas, es frecuente la inducción del trabajo de parto una vez que se diagnostique la muerte; a pesar de ello, Weeks ${ }^{1}$ menciona "aunque se han descrito muchos regímenes diferentes de inducción, ningún método de inducción ha sido estudiado rigurosamente. Además, la mayoría de los datos sobre el método de inducción se han extrapolado de la inducción del trabajo de parto en embarazos con un feto viable o de la terminación de los embarazos en el segundo trimestre. Estas circunstancias son diferentes de la inducción de un nacido muerto y pueden no ser aplicables".

Por tanto, de acuerdo con el aporte de los autores en su estudio, a partir del surgimiento de métodos farmacológicos como inductores que pueden ser utilizados en el manejo de la muerte fetal, es más frecuente que, una vez diagnosticada dicha condición, se proceda a inducir el parto; no obstante, el estudio apunta a la ausencia de un régimen de inducción que sea estudiado con rigurosidad y a partir del cual la evidencia recomiende la mejor opción por seguir en el manejo de la oxitocina en muerte fetal (para interés de la presente investigación) tomando en consideración las características específicas de cada mujer. A pesar de que otros métodos han sido estudiados 
más minuciosamente (por ejemplo, el misoprostol), los estudios se orientan hacia el tiempo del parto desde el inicio de la inducción.

Dado lo anterior, se brinda un acercamiento sobre un punto controversial en cuanto al método de inducción como lo es el uso y administración de la oxitocina en mujeres que presentan una muerte fetal, a raíz del cuestionamiento de las investigadoras sobre las dosis óptimas que ofrezcan eficacia y seguridad a la madre, a quien se le debe brindar una atención que garantice su bienestar, mediante una práctica segura; por tanto, al no encontrarse referencias que respalden ésta práctica, de acuerdo con la búsqueda realizada en más de 2000 artículos científicos de diferentes bases de datos científicas, se consulta en la literatura sobre las dosis de oxitocina recomendadas para la inducción de fetos viables. Al respecto en el estudio de Dupont et $\mathrm{al}^{21}$, refieren que no hay base científica para concluir sobre las dosis óptimas de eficacia de la oxitocina. No se recomienda administrar oxitocina a una dosis inicial mayor o igual a $4 \mathrm{mUI} / \mathrm{min}$ (Grado B), sino comenzar con una dosis inicial de $2 \mathrm{mUI} / \mathrm{min}$ (acuerdo profesional). Se aconseja observar intervalos de al menos 30 minutos antes de cada aumento de dosis de oxitocina (grado B). No hay base científica para concluir que la dosis óptima de nivel es apropiada. Se propone aumentar la oxitocina por dosis de $2 \mathrm{mIU} / \mathrm{min}$, sin exceder una tasa absoluta de $20 \mathrm{mIU} / \mathrm{min}$, y dejar de aumentar las dosis tan pronto como las contracciones uterinas tengan una frecuencia de 5 durante $10 \mathrm{~min}$ (acuerdo profesional).

Complementando lo expuesto, en el estudio seleccionado y sometido a análisis realizado por Budden, Chen, Henry ${ }^{19}$ en el que se comparó dosis altas de oxitocina versus bajas, se definió como protocolo la dosis alta de oxitocina de al menos $100 \mathrm{mU}$ en los primeros 40 minutos, con incrementos de al menos $600 \mathrm{mU}$ en las dos primeras horas, en comparación con la dosis baja de oxitocina, definida como menos de $100 \mathrm{mU}$ de oxitocina en los primeros 40 minutos, y los incrementos de entrega de menos de $600 \mathrm{mU}$ total en las dos primeras horas. A pesar de los resultados arrojados sobre factores como la hiperestimulación uterina, rotura uterina, hemorragia posparto, morbilidad y mortalidad materna, entre otros, se recomienda más investigaciones, puesto que la evidencia actual no es lo suficientemente fuerte para apoyar la administración de dosis altas sobre dosis bajas de oxitocina en la inducción del parto.

De igual forma, Dupont et al también aportan que "la administración de oxitocina durante el trabajo de parto espontáneo se asocia con un mayor riesgo de hemorragia postparto, especialmente cuando la dosis utilizada durante el trabajo de parto es alta, si los intervalos de aumento son cortos, si el paciente no se beneficia de la administración profiláctica de oxitocina durante el parto (NP3). Parece que hay una asociación entre la oxitocina y la rotura uterina en el útero sano (NP4) ${ }^{21}$."

\section{CONCLUSION}

Con el proceso de evaluación de la evidencia científica disponible la aplicación de dosis altas de oxitocina versus dosis estándar para la inducción del parto en muerte fetal y el riesgo de complicaciones y muerte materna, se encuentró que la evidencia científica sobre los métodos de inducción en mujeres con muerte fetal está orientada a la comparación entre los diferentes métodos, basados principalmente en el tiempo desde la inducción hasta el parto. De esta manera, específicamente con la administración de oxitocina, las dosis por utilizar, así como el efecto sobre la madre, se llega a la conclusión final de que no se encontraron estudios específicos para analizar el manejo más adecuado para la inducción de parto en caso de muerte intrauterina. 
Declaración de conflicto de intereses. Las investigadoras declaran que no tienen ningún conflicto de intereses de índole personal, social, económico ni laboral.

\section{REFERENCIAS BIBLIOGRÁFICAS}

1.Weeks A. Tratamiento clínico para la muerte fetal temprana (menos de 24 semanas): Comentario de la BSR). La Biblioteca de Salud Reproductiva de la OMS; Ginebra: Organización Mundial de la Salud. 2008. (última revisión: 4 de enero de 2007) Disponible en: https://extranet.who.int/rhl/es/topics/pregnancy-andchildbirth/antenatal-care/miscarriage-1

2.Organización Mundial de la Salud. Mortalidad Materna. Nota descriptiva N³48 noviembre de 2015. Disponible en: http://www.who.int/mediacentre/factsheets/fs348/es

3.Reyna E, Guerra M, Montilla M, Reyna N, Mejía J. Misoprostol oral u oxitocina para la expulsión del Feto Muerto Intrauterino en el segundo trimestre. Revista de Facultad de Medicina, Universidad de Los Andes (Internet). 2005.13 (1-4): 29-32. Disponible en: http://erevistas.saber.ula.ve/index.php/medula/article/view/5713

4.Gawron LM, Kiley JW. Labor induction outcomes in third-trimester stillbirths. International Journal of Gynecology and Obstetrics. (internet) 2013.Dec; 123 (3)203-6. Disponible en: https://www.ncbi.nlm.nih.gov/pubmed/24059984

5.Grotegut C, Paglia M, Johnson L, Thames B, \& James, A. Oxytocin exposure during labor among women with postpartum hemorrhage secundary to uterine atony. Revista Americana de Ginecología y Obstetricia (internet) 2011. 204 (56) 1-6. Disponible en: https://www.ncbi.nlm.nih.gov/pubmed/21047614

6.Abha Singh, Chandrashekhar Shrivastav. Uterine Rupture: Still a Harsh Reality. J Obstet Gynaecol India. (internet) 2015 May; 65(3): 158-161. Disponible en: https://www.ncbi.nlm.nih.gov/pmc/articles/PMC4464568/

7. Ladewing P, London M, Moberly S, Olds S. Enfermería Maternal y del recién nacido. Editorial McGraw-Hill interamericana. España. 2006

8. Schwarcz R, Fesina R, Duverges C. Obstetricia. Editorial el Ateneo. Sexta Edición. Buenos Aires. 2012

9.Alcolea M, Oter C, García A. Fases de la Práctica Basada en la Evidencia. Nurse Investigación N 53. 2011. Disponible en: http://www.nureinvestigacion.es/OJS/index.php/nure/article/download/538/52

10.Muñoz C, Munar J, González H, López A. (2012). Actualización en el uso de uterotónicos. Rev Esp Anestesiol Reanim. 2012;59(2):91-97. Disponible en: http://www.elsevier.es/es-revista-revista-espanola-anestesiologiareanimacion-344-articulo-actualizacion-el-uso-uterotonicos-S0034935612000527 


\section{Revista Electrónica Enfermeria Actual en costa Rica}

11.González J, Buñuel JC, Ochoa C. La valoración crítica de documentos científicos y su aplicabilidad a la práctica clínica: aspecto clave en la toma de decisiones basada en las mejores pruebas científicas. Evid Pediatr. [Internet] 2006 [acceso 20 noviembre de 2016]; (2): 37. Disponible en:

http://www.evidenciasenpediatria.es/articulo/5047/la-valoracion-critica-de-documentos-cientificos-y-suaplicabilidad-a-la-practica-clinica-aspecto-clave-en-la-toma-de-decisiones-basada-en-las-mejores-pruebascientificas

12. Cabero L; Saldivar D; Cabrillo E. Obstetricia y medicina Materno fetal. Editorial Médica Panamericana. Madrid. 2007.Disponible en:

https://books.google.co.cr/books?id=AGh8rK1MmOsC\&pg=PA60\&dq=definición+de+autonom\%C3\%ADa+co $\underline{m o}+$ consideracion + etica\&hl $=$ es-

419\&sa $=X \&$ redir_esc $=y \# v=$ onepage $\& q=$ definición $\% 20 \mathrm{de} \% 20$ autonom $\% \mathrm{C} 3 \% \mathrm{ADa} \% 20$ como $\% 20$ consideracion $\%$ 20etica\&f $=$ false

13. Siruana J. Los principios de la bioética y el surgimiento de una bioética intercultural. Revista veritas (internet) 2010. Acceso marzo 2010. 22, 121-157. Disponible en: http://www.scielo.cl/pdf/veritas/n22/art06.pdf

14. De la Torre FJ. Ética y Deontología Jurídica. Editorial Dykinson. Madrid. 2000. Disponible en: http://www.worldcat.org/title/etica-y-deontologia-juridica/oclc/45345700

15. Redondo JA. Creando clientes en mercados globales $=$ Building client relationships in global markets Editorial AEDEM. Madrid. 2010. Disponible en: http://www.worldcat.org/title/creando-clientes-en-mercadosglobales-building-client-relationships-in-global-markets/oclc/746499001

16.Laguna S, Caballero C, Lewis V, Mazuera S, Salamanca J, Daza W y Fourzali A (2007). Consideraciones éticas en la publicación de investigaciones científicas. Salud Uninorte.23 (1): 64-78. Disponible en: http://www.scielo.org.co/pdf/sun/v23n1/v23n1a08

17.Normas Vancouver. Citas y Referencias Bibliográficas según Normas Vancouver. 2014. Disponible en:

https://es.slideshare.net/BibliotecasUNAB/normas-vancouver-2016-sistema-bibliotecas-unab

18. Sésgales J. Sobre la Declaración de Conflictos de Interés. Rev Cient Cienc Méd. v.14 n.1 Cochabamba 2011. versión impresa ISSN 2077-3323

19.Budden A, Chen LJY, Henry A. High-dose versus low-dose oxytocin infusion regimens for induction of labour at term. Base de Datos Cochrane de Revisiones Sistemáticas. 2014, Número 10. Disponible en: http://www.cochrane.org/CD009701/PREG high-dose-versus-low-dose-oxytocin-infusion-regimens-inductionlabour 
UNIVERSIDAD DE

COSTARICA

| Edición Semestral Nº. 34, Enero 2018 - Junio 2018 | ISSN 1409-4568 |

Revista Electrónica Enfermería Actual en costa Rica

www.revenf.ucr.ac.cr

20.Boyle A, Preslar J, Hogue C, Silver R, Goldenberg R, Stoll B, et al. Delivery in Women with Stillbirth: Results from the Stillbirth Collaborative. Research Network. The American College of Obstetricians and Gynecologists. 2017. Apr; 129 Apr;129(4):693-698 Disponible en:

https://www.ncbi.nlm.nih.gov/pubmed/28277361

21.Duponta C, Carayolc M, Le Rayd C, Barasinskif C, Berangerg R, Burgueth A et. al. Recommandations pour 1' administration d'oxytocine au cours du travail spontané. Texte court des recommandations. Gynécologie Obstétrique Fertilité \& Sénologie (2017); 45(1):56-61. Disponible en:

http://doi.org.ezproxy.sibdi.ucr.ac.cr:2048/10.1016/j.gofs.2016.12.017

Revista Enfermeria Actual, teléfono 2511-21-18 (Escuela de Enfermeria), fax 2511-4775. Dirección: Ciudadela Rodrigo Facio,

Ciudad_de_la_Investioación_contioun_aLINIISA_Sahanilla_de_Montes_de_Oca_San_losé_Costa_Rica 